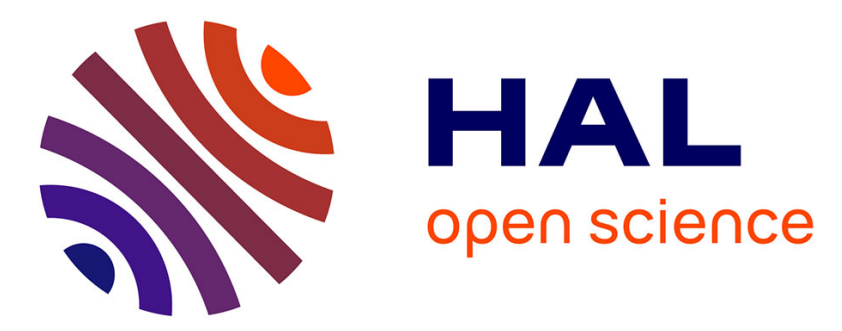

\title{
Bulk supercooled water versus adsorbed films on silica surfaces: specific heat by Monte Carlo simulation
}

\author{
Joël Puibasset, Patrick Judeinstein, Jean-Marc Zanotti
}

\section{To cite this version:}

Joël Puibasset, Patrick Judeinstein, Jean-Marc Zanotti. Bulk supercooled water versus adsorbed films on silica surfaces: specific heat by Monte Carlo simulation. Physical Chemistry Chemical Physics, 2021, 10.1039/D0CP05387B . hal-03108590

\section{HAL Id: hal-03108590 \\ https://hal.science/hal-03108590}

Submitted on 13 Jan 2021

HAL is a multi-disciplinary open access archive for the deposit and dissemination of scientific research documents, whether they are published or not. The documents may come from teaching and research institutions in France or abroad, or from public or private research centers.
L'archive ouverte pluridisciplinaire HAL, est destinée au dépôt et à la diffusion de documents scientifiques de niveau recherche, publiés ou non, émanant des établissements d'enseignement et de recherche français ou étrangers, des laboratoires publics ou privés. 


\section{Bulk supercooled water versus adsorbed films on silica surfaces: specific heat by Monte Carlo simulation}

Received 00th January 20xx, Accepted 00th January 20xx DOI: $10.1039 / \times 0 \times x 00000 x$

\author{
J. Puibasset, ${ }^{* a}$ P. Judeinstein ${ }^{\mathrm{b}}$ and J.-M. Zanotti ${ }^{\mathrm{b}}$
}

Between 150 and $230.6 \mathrm{~K}$, bulk supercooled water freezes upon cooling, and amorphous ice crystallizes upon heating: bulk water thus exists only in its stable ice form. To circumvent this problem, experiments are generally performed on water adsorbed in $\mathrm{SiO}_{2}$ based porous systems. In this work, we take advantage of Monte Carlo simulations to explore this metastable supercooled region inaccessible to experiments. Using three rigid, non-polarizable water models, namely SPC, TIP4P and TIP4P/2005, we investigate the isobaric specific heat capacity $\left(C_{p}\right)$, between 100 and $300 \mathrm{~K}$, of bulk water and water films of few monolayers adsorbed on different $\mathrm{SiO}_{2}$ surfaces: a smooth surface, a non-hydroxylated (0001) surface of quartz, and a fully hydroxylated (001) surface of cristobalite. As $C_{p}$ is directly related to the entropy fluctuations and we focus on low temperatures, the convergence of the Monte Carlo simulations is a critical point of this work. Also, due to the small mass of the hydrogen atoms, quantum corrections are taken into account, and lead to an excellent agreement of the simulated and experimental $C_{p}$ values at low temperature ( $100 \mathrm{~K}$ region). Altogether, we conclude that, in bulk, $C_{p}$ is shown to exhibit a broad peak around $225 \mathrm{~K}$ for the SPC and TIP4P models, and around $250 \mathrm{~K}$ for the TIP4P/2005 model, in qualitative agreement with the experimentally observed features in $C_{p}$ measurements. For interfacial water, in all cases, the broad $C_{p}$ peak disappears. This result, at odd with experimental observations, suggests that disorder and hydrogen bonding at the interface (not yet taken into account) have a fundamental role in confined water transitions.

\section{Introduction}

Several thermodynamic anomalies in supercooled water are still a matter of debate. ${ }^{1-19}$ Careful analysis of experimental data in the 1970s showed a sharp increase upon cooling of thermodynamic response functions of water, like its isothermal compressibility or the isobaric heat capacity. ${ }^{20}$ These quantities exhibit apparent critical power-law divergence at $228 \mathrm{~K}$. Bulk water has a very specific tetrahedral organization of its hydrogen bond network, which is believed to be closely connected to the existence of the observed specific properties of water, like the existence of a temperature of maximum density or a possible liquid-liquid transition in the supercooled region associated with a second critical point at $228 \mathrm{~K}$ and 100 MPa. 2, 5, 6

Unfortunately, experimentally, upon cooling, bulk liquid (supercooled) water spontaneously freezes below the homogeneous nucleation temperature $(230.6 \mathrm{~K}){ }^{21}$ while upon heating, amorphous solid water crystallizes above $150 \mathrm{~K}$. The so-called no man's land between 150 and $230.6 \mathrm{~K}$ is thus inaccessible to experiments. In this context, a number of research works proposed to consider the "confined water"

a.ICMN, UMR7374, CNRS, Université d'Orléans, 1b, rue de la Férollerie, 45071,

Orléans cedex 2, France. E-mail : puibasset@cnrs-orleans.fr

b. Université Paris-Saclay, CEA, CNRS, LLB, 91191, Gif-sur-Yvette, France. adsorbed in silica-based materials (MCM41, Vycor...) as a promising model of the physics of supercooled liquid water in the no man's land. ${ }^{6}, 13,14,16,22-25$ In the case of Vycor, the high density of silanols at the Vycor surface $\left(16 \mathrm{OH} / \mathrm{nm}^{2}\right)$ enables a similarity between a water monolayer at the interface and the bulk water. ${ }^{22}$ Several "water anomalies" show up through different experimental techniques (calorimetry, neutron, NMR, near and far infrared spectroscopies) around 150, 220 and 250 $\mathrm{K}$, which appear as new signals/species or through the modification of their frequency, linewidth or intensity. $22,26,27$ Thermal events are also detected around these temperatures in heat exchange measurements. ${ }^{23,} 28-34$

These water anomalies are generally associated with structural or dynamical transitions of water. Recent studies have focused on the case of interfacial situations where the water molecules are forced to organize in only two dimensions. ${ }^{33}, 35$ This profoundly affects the physics of water, and rises questions regarding the role of the hydrogen bonding of the first water layer with the silica and its hydroxylation state. As a general result, it is well known that confinement may remove some of water thermodynamic anomalous properties. ${ }^{36}$ This issue is of uppermost importance regarding water properties in the no man's land, since it is impossible to have a clear answer from the experimental side solely. Theoretical investigations are thus mandatory to interpret experimental data: is interfacial water a good model of supercooled water or not? No clear-cut interpretation of the phenomenon has been reached so far, which has stimulated this numerical study. 
Computer simulation is widely used to provide important information on the structure of water at the molecular level, as well as its dynamical and thermodynamic properties. The quality of the intermolecular potential is of course mandatory for reliable results. A number of different potential models have been proposed, the simplest being rigid with fixed partial charges, to more sophisticated ones including flexible bonds and polarizability. ${ }^{37,}{ }^{38}$ No water model is totally satisfactory, even if the differences between water models are not that huge. Our interest being the physical properties of the supercooled phase, it is mandatory to focus on fast models in terms of computational cost, in particular rigid, non-polarizable models. One of the most popular is probably the Simple Point Charge (SPC) model, ${ }^{39}$ which has been intensively used in molecular simulations involving large systems. The second very popular model is the Transferable Intermolecular Potential TIP4P. 40 These models have been shown to be in reasonable agreement with experiments, regarding the structure, the dynamics and thermodynamic quantities like the enthalpy or the heat capacity, of interest for us. ${ }^{37,}{ }^{41}$ However, the phase diagram was not quantitatively reproduced, and this has stimulated the development of the TIP4P/2005 model by Abascal and Vega. ${ }^{42}$

Nanoporous silica has a great ability to adsorb water, and the ubiquity of this system has prompted many studies by molecular simulations. ${ }^{43-52}$ It is well-known that the chemical and morphological disorder of the adsorbing surface plays a major role in adsorption. ${ }^{53-58}$ An important issue of water adsorbed on hydroxylated silica surfaces concerns the connection between the three dimensional hydrogen bond network belonging to water with the two-dimensional $\mathrm{H}$-bonds at the water-silica interface. This is expected to have an impact on the heat capacity of adsorbed layers of water through the local dynamics of adsorbed molecules. The objective of this paper is to present heat capacity calculations of bulk water and water films adsorbed on different flat hydrophilic surfaces, in order to evidence the effect of surface roughness or hydroxylation.

\section{Model and Methods}

\section{Models and interactions}

We have performed molecular Monte Carlo simulations of water in order to calculate its heat capacity in different situations. We have considered three widely used models: SPC, ${ }^{7}$ TIP4P, 40 and TIP4P/2005. ${ }^{42}$ These models are rigid and nonpolarizable: they thus represent a simplified version of the true interactions in water. In the SPC model, the $\mathrm{OH}$ bond length is 1 $\AA$ and the $\mathrm{H}-\mathrm{O}-\mathrm{H}$ angle is $109.47^{\circ}$ (the tetrahedral value). In the TIP4P and TIP4P/2005 models, the $\mathrm{OH}$ bond length and $\mathrm{H}-\mathrm{O}-\mathrm{H}$ angle are those of the molecule in the gas phase, namely 0.9572 $\AA$ and $104.52^{\circ}$ respectively. Note that these models do not take into account important quantum nuclear effects due to the hydrogen species: these will be added as corrections for the heat capacity calculations. ${ }^{37}$ More details are given below. A full quantum mechanical treatment of water would be impossible at low temperature in the supercooled region due to the dramatic slowing down of the dynamics, while classical models are fast enough to enable accurate simulations in the low temperature regime. The drawback of this approach is that the molecular structure and trajectories cannot be corrected for quantum effects.

The charge distribution in water models is generally represented by point charges on the nuclei, and the dispersion forces are located on or close to the oxygen species. The SPC model is a three-site model (the dispersion forces are located on the oxygen) and the TIP4P and TIP4P/2005 are four-site models (the dispersion force is located on the so-called $\mathrm{M}$ site at a distance of $0.15 \AA$ on the $\mathrm{H}-\mathrm{O}-\mathrm{H}$ bisector. The parameters of the models have been determined in order to reproduce the liquid water density, the heat of vaporization at room temperature or the phase diagram of ice. ${ }^{37,40-42}$ The tetrahedral structure of water is also reasonably reproduced by SPC, while a better agreement is obtained with TIP4P. From a dynamical point of view, the SPC model diffusion coefficient (or rotational constant) are too fast by a factor two, those obtained from TIP4P are only $50 \%$ faster and TIP4P/2005 is quite accurate, i.e. slightly below the experimental data and with the correct trend in its dependence with temperature. ${ }^{37}, 59$ Water exhibits a maximum density around $4^{\circ} \mathrm{C}$, closely connected to the hydrogen bond network. This is reproduced by TIP4P and TIP4P/2005 models, but not by the SPC model. The phase diagram is also a stringent test of the accuracy of a water model. It has been found that SPC is not able to reproduce qualitatively the phase diagram of water, while TIP4P provides a qualitatively correct phase diagram and TIP4P/2005 appears quite accurate, in particular regarding the solid/liquid transition for the ice Ih. ${ }^{59}$ The calculations are done by Monte Carlo using the Metropolis algorithm. For the bulk water calculations, the temperature and pressure are controlled (isobaric-isothermal NPT ensemble), while for the water films grown on the surfaces, the calculations are done in the canonical ensemble. These ensembles are chosen to reproduce the experimental situations, namely constant pressure-temperature measurements for bulk water, and liquid-vapour equilibrium for water films adsorbed on a surface. Note that in the latter case, the grand canonical ensemble could also be used, with an imposed chemical potential corresponding to the desired film thickness. It is however simpler to work in the canonical ensemble so that the film thickness can be imposed without the need for the calculation of the adsorption isotherm. This is particularly useful if one wants to compare two systems with different surface chemistry (and thus adsorption affinity) but identical adsorbed film thicknesses.

The systems were simulated in a wide range of temperatures, between 100 and $300 \mathrm{~K}$. Below the melting temperature, the water can either form a crystalline structure (ice $\mathrm{lh}$ ) or remain in the metastable supercooled phase due to the large barriers to nucleate the crystalline form. In the latter case, for a sufficiently low temperature, the liquid-to-solid transition becomes experimentally unavoidable. However, in simulations, the transition is never observed due to large energetic barriers, strong slowdown of the dynamics, and comparatively limited 
simulation runs. It is then easy to observe in simulations the metastable supercooled phase down to $100 \mathrm{~K}$ (which forms an amorphous solid at such a low temperature). Of course, it is desirable to compare the simulation results for the metastable supercooled phase with those expected for the stable crystalline phase. This is why these two phases have been numerically produced as follows.

(i) The metastable liquid or amorphous solid (undistinguishable in our simulations) are obtained by progressively cooling down an initial configuration corresponding to the liquid state above the melting temperature. Starting from the liquid water at $\mathbf{3 0 0}$ $\mathrm{K}$, the temperature is decreased by steps of $25 \mathrm{~K}$, the final configuration of each run being used as the initial configuration for the next temperature. This procedure is thought to be the most efficient to equilibrate the system at low temperatures, where the mobility of the molecules is extremely low. This recipe has been applied for the bulk water as well as for the water films adsorbed on a surface.

(ii) For the bulk crystalline ice phase, the simulations are started from the lowest temperature $(100 \mathrm{~K})$ with an initial configuration corresponding to the ideal hexagonal structure $\mathrm{lh}$. The temperature is then increased by steps of $25 \mathrm{~K}$, and as before, the final configuration for a given temperature is used as the initial configuration of the next temperature, in order to optimize the Monte Carlo convergence.

Table 1: Dimensions of the simulation box $(\mathrm{nm})$, number of water molecules (two values are considered for the three surfaces), and water surface densities (micromol $/ \mathrm{m}^{2}$ ).

\begin{tabular}{|c|c|c|c|c|c|}
\hline & $\begin{array}{c}\mathbf{L}_{\mathbf{x}} \\
(\mathbf{n m})\end{array}$ & $\begin{array}{c}\mathbf{L}_{\mathbf{y}} \\
(\mathbf{n m})\end{array}$ & $\begin{array}{c}\mathbf{L}_{\mathbf{z}} \\
(\mathbf{n m})\end{array}$ & nb $\mathrm{H}_{2} \mathrm{O}$ & $\begin{array}{c}\text { surface } \\
\text { density } \\
\left(10^{-6} \mathrm{~mol} / \mathrm{m}^{2}\right)\end{array}$ \\
\hline bulk liquid & 1.86 & 1.86 & 1.86 & 216 & -- \\
\hline bulk Ice Ih & 1.8 & 2.35 & 2.2 & 288 & -- \\
\hline smooth surface & 3.725 & 3.725 & 4.00 & $220 / 440$ & $26 / 52$ \\
\hline quartz (0001) & 3.931 & 3.404 & 4.00 & $212 / 424$ & $26 / 52$ \\
\hline cristobalite (001) & 3.583 & 3.583 & 4.00 & $203 / 406$ & $26 / 52$ \\
\hline
\end{tabular}

The simulations of the bulk liquid and metastable amorphous phases are done using a cubic box containing 216 molecules with periodic boundary conditions. The lateral dimensions are allowed to fluctuate (isobaric ensemble), their average value being close to $\mathrm{L}=1.86 \mathrm{~nm} .{ }^{60}$ The bulk ice $\mathrm{lh}$ simulations are done using a box as close as possible to the previous one, taking into account the constraints imposed by the crystallographic parameters of ice. The initial dimensions are approximately 1.8 , 2.35 and $2.2 \mathrm{~nm}$ along the $\mathrm{x}, \mathrm{y}$ and $\mathrm{z}$ directions, with 288 water molecules and periodic boundary conditions. The water films are studied in larger simulation boxes, whose precise dimensions depend on the nature of the substrate. More details are given below and in Table 1 . In all cases, the substrate is parallel to the $x$ and $y$ directions (see Fig. 1a for a general view). The dimension of the simulation box in the $z$-direction is chosen large enough compared to the thickness of the silica surface plus its adsorbed water film in order to have an empty space above the film that is sufficiently large $(>1 \mathrm{~nm})$ to avoid any disturbance due to the application of the periodic boundary conditions. Note that the number of water molecules is kept constant during these simulations (canonical ensemble). It is however important to notice that the empty space above the film allows its equilibration with its own vapour. In practice, the vapour pressure being very low below $300 \mathrm{~K}$, vapour molecules are scarcely observed during the simulations.
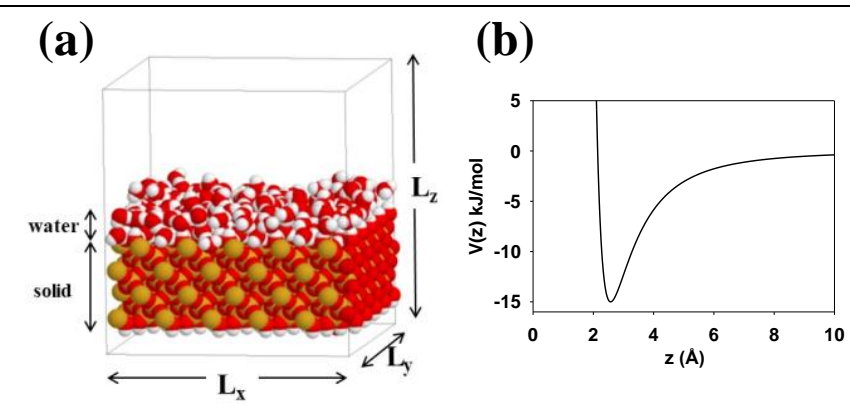

(c)

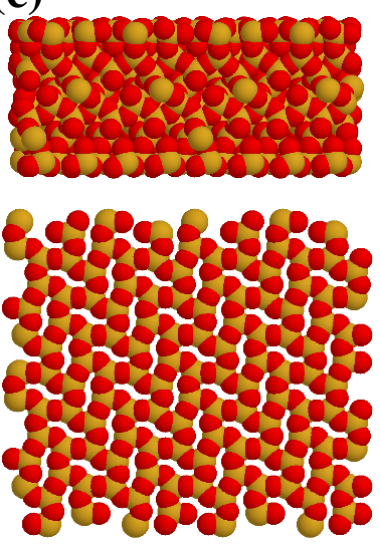

(d)
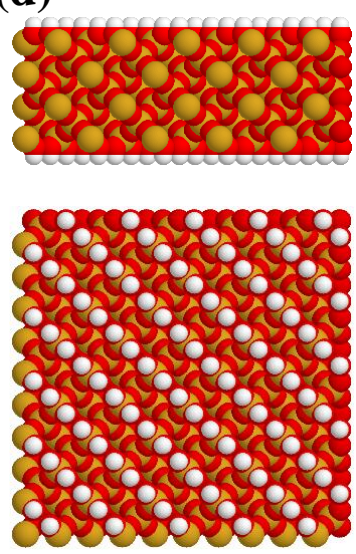

Fig. 1. (a) Schematic representation of the simulation box showing the surface parallel to the $x$ and $y$ axes, and the water film adsorbed on the upper face. The lower face is free of molecules. The three surfaces used in this work are: (b) the smooth-wall approximation, (c) the anhydrous quartz (0001) and (d) the fully hydroxylated cristobalite (001).

The water-water contribution to energy is calculated with minimal image convention, with a cutoff for the Lennard-Jones (U) contribution equal to $0.9 \mathrm{~nm}$. Long-range corrections are neglected for the $\mathrm{L}$ term. However, long-range electrostatic contributions are handled with the Ewald summation. The direct space parameter kappa and the number of reciprocal vectors are chosen to be respectively $2.288 \mathrm{~nm}^{-1}$ and $7^{3}$. The total expected precision is around $1 \%$. The interactions between the water molecules and the atoms of the silica surface contain van der Waals and electrostatic contributions due to the hydroxyls at the silica surface. These interactions are described with the Brodka and Zerda potential. ${ }^{61}$ 


\section{Silica surfaces}

The objective of this study is to provide a molecular model able to describe the experimental behaviour of the heat capacity of water adsorbed on silica surfaces. ${ }^{35}$ The film configurations thus correspond to water adsorbed on hydrophilic surfaces. The surface roughness is expected to be an important parameter since the structure of water at the interface is largely influenced by the local morphology of the surface. We thus consider, on the one hand, a surface where the atomic roughness is smoothed out, and, on the other hand, atomistic surfaces. It is also desirable to evidence the specific contributions of the surface hydroxyl groups: we will thus consider surfaces with and without hydroxyls.

Smooth surface. In the smooth wall approximation, the external potential felt by a water molecule adsorbed on the surface is that produced by a half-space continuum of silica species interacting with the water oxygen via for instance the 12-6 Lennard-Jones contribution of the Brodka and Zerda potential. ${ }^{61}$ The integration results in the 9-3 potential.

$V(z)=\frac{4}{3} \pi \varepsilon_{\mathrm{sf}} \rho_{\mathrm{s}} \sigma_{\mathrm{sf}}^{3}\left[\frac{\sigma_{\mathrm{sf}}^{9}}{15 z^{9}}-\frac{\sigma_{\mathrm{sf}}^{3}}{2 z^{3}}\right]=3 \sqrt{\frac{2}{5}} \varepsilon\left[\frac{\sigma_{\mathrm{sf}}^{9}}{15 z^{9}}-\frac{\sigma_{\mathrm{sf}}^{3}}{2 z^{3}}\right]$

where $\rho_{\mathrm{s}}$ is the volume density of the solid interaction sites, and $\varepsilon_{\mathrm{sf}}$ and $\sigma_{\mathrm{sf}}$ are the parameters of the 12-6 Lennard-Jones interaction between water molecules and silica. However, the external potential obtained with this procedure neglects the important contribution from the surface dipoles associated with the hydroxyls. It is therefore necessary to enhance the external potential so that the minimum equals $-15 \mathrm{~kJ} / \mathrm{mol}$, representative of a hydrophilic silica surface, while keeping the same functional form. ${ }^{43}, 44$ The potential is thus given by the right-hand side of eqn (1) with $\varepsilon=15 \mathrm{~kJ} / \mathrm{mol}$ (see Fig. 1b). It has been shown that this simple model catches the main features of a hydrophilic surface, in particular in terms of water adsorption: it reproduces the adsorption isotherm obtained by taking into account explicitly the electrostatic contributions due to the partial charges of the silica species, in particular the presence of hydroxyls. ${ }^{62,63}$

Quartz surface. The second surface to be considered is the (0001) face of $\alpha$-quartz. This surface is quite stable and has been extensively studied in experiments and theoretically. The native (0001) surface undergoes a reconstruction depending on the temperature. In particular, $1 \times 2$ reconstructions have been observed through DFT and classical MD calculations that can explain experimental observations. ${ }^{64,65}$ These reconstructions are stable at room temperature and below and have been chosen for our model (see Fig. 1c). Note that this surface may be completely dehydroxylated, and will be used as an example of surface with atomic roughness but without hydroxyl groups. The absence of surface dipoles $(-\mathrm{OH})$ has consequences on the adsorption of water (less hydrophilic), but this is partially compensated by the fact that the first atomic layer in (0001) quartz is much denser than silica.
Cristobalite surface. The surface density of hydroxyl groups on silica depends on the conditions of preparation, like the presence of air, and the temperature. The typical average surface density encountered in experiments is around 5 $\mathrm{OH} / \mathrm{nm}^{2}{ }^{66}$ A simple model of hydroxylated silica surface may then be obtained by fully saturating with hydrogen the (001) surface of $\beta$-cristobalite, giving a surface density of $4.5 \mathrm{OH} / \mathrm{nm}^{2}$ (see Fig. 1d). It has been shown that this surface is able to reproduce the adsorption isotherm of water on silica. ${ }^{62}$.

\section{Water films and heat capacity calculations}

The water films were initially obtained on the smooth surface following the grand canonical Monte Carlo procedure as previously described. ${ }^{63,67}$ Two film thicknesses have then been chosen, that correspond approximately to one and two water layers. These grand canonical calculations have been performed on the smooth surface only; for the other surfaces, the number of molecules was rescaled according to the area in order to have the same film thickness in all samples (see Table 1 ).

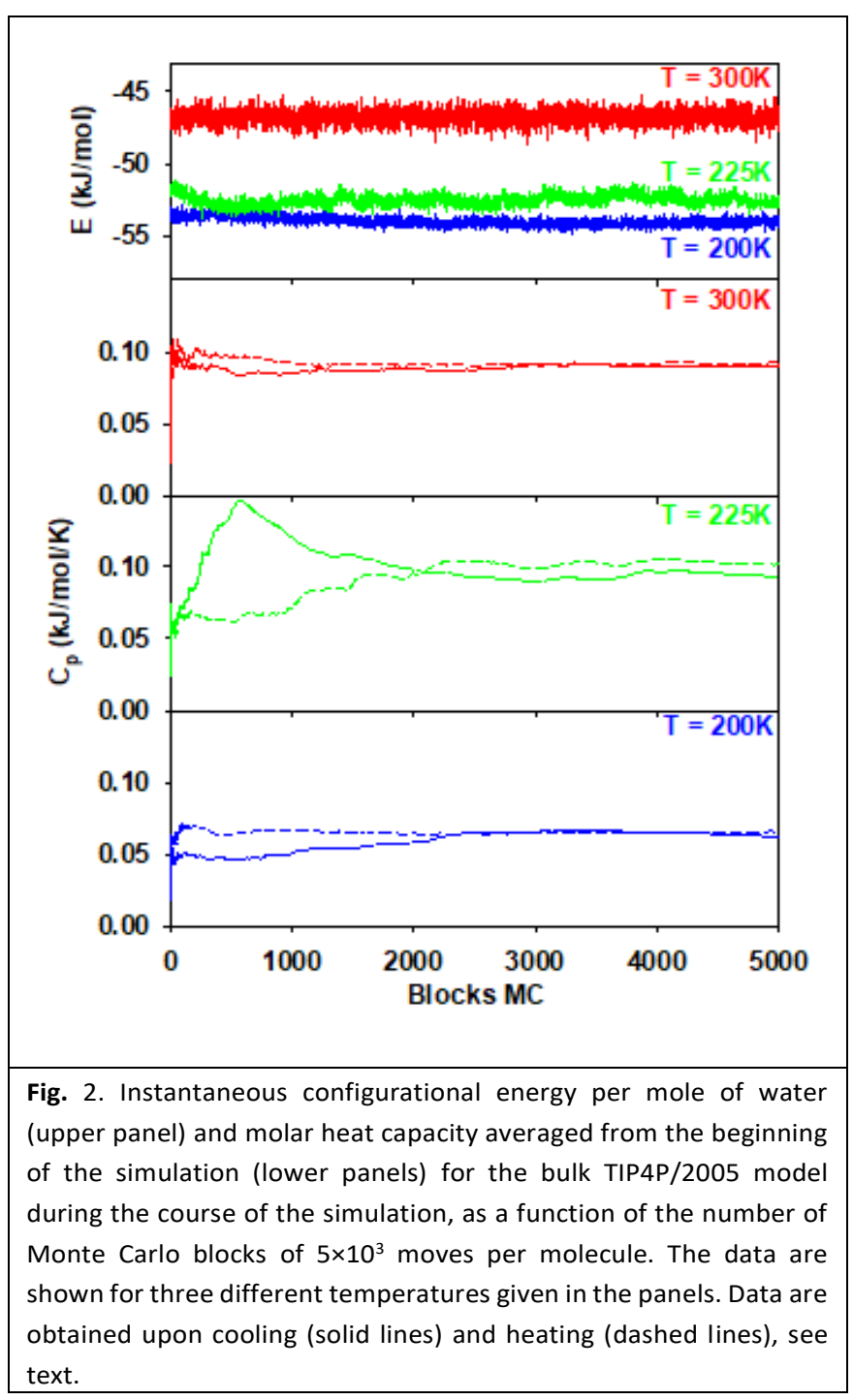


The heat capacity of bulk water and ice are calculated in the isobaric-isothermal ensemble, and for water films, they are calculated in the canonical ensemble. In the latter case, the system contains a liquid/vapour interface, which is free to move to equilibrate pressure and chemical potential between the liquid film and the vapour. This follows exactly the experimental situation where the porous material + water system is generally sealed in vessels to avoid evaporation. The heat capacity is obtained from the standard fluctuations formula, during the Monte Carlo run, of the enthalpy $\left(\left\langle\mathrm{H}^{2}\right\rangle-\langle\mathrm{H}\rangle^{2}\right) / \mathrm{NkT}^{2}$ in the isothermal-isobaric ensemble, or energy $\left(\left\langle\mathrm{E}^{2}\right\rangle-\langle\mathrm{E}\rangle^{2}\right) / \mathrm{NkT}^{2}$ in the isothermal-isochoric ensemble. ${ }^{68}$

\section{Convergence of the Monte Carlo calculations}

The important point of the convergence of the Monte Carlo calculations is addressed now. It is well known that the calculation of the heat capacity is computing time demanding. ${ }^{60}$ Long Monte Carlo runs were performed to reach the equilibrium: $5 \times 10^{6}$ Monte Carlo steps per molecule for each temperature for equilibration, followed by $25 \times 10^{6}$ steps per molecule for data acquisition. Fig. 2 shows the configurational energy and the heat capacity per water mole of the TIP4P/2005 model during the course of the simulation as a function of the number of blocs of $5 \times 10^{3}$ Monte Carlo steps per molecule, and for three temperatures $(300,225$ and $200 \mathrm{~K}$ ) chosen along the descending temperature ramp (solid lines). One observes a fast convergence of the simulations in terms of energy, while the heat capacity exhibits large variations up to 2 to 3 thousands Monte Carlo blocks. Furthermore, the energy is an instantaneous quantity while the heat capacity is derived from a fluctuation formula that takes into account all data from the beginning: as a consequence, the error bars in energy are given by the fluctuations divided by the square root of the number of independent data, while the errors for the heat capacity derive directly from the fluctuations in the last data. It is important to observe in Fig. 2 that the convergence depends on the temperature. The best convergence is obtained for the highest temperature $(300 \mathrm{~K})$, while large fluctuations appear at $225 \mathrm{~K}$ with correspondingly large error bars. At $200 \mathrm{~K}$, the fluctuations are smaller, with an amplitude similar to what is observed at $300 \mathrm{~K}$. This apparent better convergence has to be tempered by the fact that the dynamics of the system is slowed down at low temperature, which consequently decreases the apparent fluctuations that can be observed on a limited Monte Carlo run. To circumvent this problem, it is possible to evaluate the convergence by reversing the temperature ramp and comparing the new heat capacity calculations (Fig. 2, dashed lines, increasing $\mathrm{T}$ ) with the previous ones (solid lines, decreasing $\mathrm{T}$ ). A systematic difference between the two limiting values would be the signature of a drift due to a slow convergence of the Monte Carlo runs at low temperature. The differences between the two heat capacities are of the same magnitude as the error bars derived from the fluctuations for each run: this shows that a stationary regime is reached for each temperature, which does not depend on the temperature history. It is possible to draw two curves giving the heat capacity versus descending or ascending temperature: they are given in Fig. 3 (up and down triangles) for the TIP4P/2005 model. As can be seen, the differences are quite small compared with the variations of $C_{p}$ between 100 and $300 \mathrm{~K}$. Note that the errors and differences are the largest at $225 \mathrm{~K}$, without exceeding $15 \%$ of the maximal range of $C_{p}$ values. We are lead to the conclusion that the systematic errors are smaller that statistical errors, and that the curves are reversible and thus convergence is reached for each temperature. It is emphasized that we do not mean that the true thermodynamic equilibrium is reached (otherwise the stable crystalline phase would be spontaneously obtained); we mean that the variation of the temperature is sufficiently slow to allow for the relaxation of the system towards a local minimum (metastable phase). Note that at low temperature, the system reaches a glassy state with thermodynamic properties depending for instance on the thermal history. The same analysis was done for all water films adsorbed on silica surfaces and for the bulk ice Ih (except that for ice the reverse procedure starts from the highest temperature were it has not melted). In all cases, the difference between the ascending and descending curves were smaller than the error bars, leading to the same conclusion. From now on, all $C_{p}$ values are obtained by averaging the descending and ascending data to improve accuracy (circles in Fig. 3).

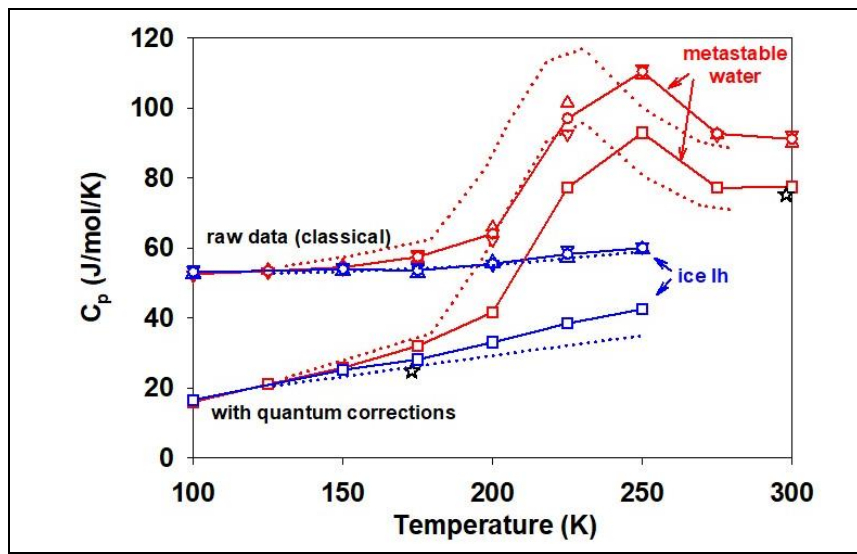

Fig. 3. Heat capacity $C_{p}$ versus temperature for the metastable supercooled water (in red) and the stable ice Ih (in blue) for the TIP4P/2005 model. Down triangles: descending temperature; up triangles: ascending temperature; circles: averaged data; squares: averaged data including quantum corrections; the solid lines are guides to the eye through the averaged data. For comparison, the MD data from Saito et al. ${ }^{78}$ are reproduced (dotted lines). Stars: experimental data for the liquid $(298 \mathrm{~K})$ and ice $(173 \mathrm{~K})$ phases. ${ }^{90-92}$

Comparison with previously published data at room temperature show good agreement. The largest differences are for the SPC model (almost 10\% variations depending on the authors) while the better agreement is for the TIP4P/2005 model (less than few percent differences). 40, 42, 59, 69-76 More specifically, for the SPC model, Wu et al. ${ }^{71}$ find at $298 \mathrm{~K} C_{p}=81.5$ $\mathrm{J} / \mathrm{mol} / \mathrm{K}$ as given by the enthalpy fluctuations formulae, and $72.2 \mathrm{~J} / \mathrm{mol} / \mathrm{K}$ after quantum corrections (see next section), which is close to our values $(84.9 \mathrm{~J} / \mathrm{mol} / \mathrm{K}$ without corrections and $71.2 \mathrm{~J} / \mathrm{mol} / \mathrm{K}$ with corrections at $300 \mathrm{~K}$ ). For the TIP4P 
model, Jorgensen et al. ${ }^{69,} 70$ give $C_{p}=91.2 \mathrm{~J} / \mathrm{mol} / \mathrm{K}$ without corrections and $83.7 \mathrm{~J} / \mathrm{mol} / \mathrm{K}$ with corrections at $298 \mathrm{~K}$. We find $89.5 \mathrm{~J} / \mathrm{mol} / \mathrm{K}$ without corrections, and $75.8 \mathrm{~J} / \mathrm{mol} / \mathrm{K}$ with corrections at $300 \mathrm{~K}$. For TIP4P/2005, Abascal et al. ${ }^{42}$ find 88.3 $\mathrm{J} / \mathrm{mol} / \mathrm{K}$ without corrections and $79 \mathrm{~J} / \mathrm{mol} / \mathrm{K}$ with corrections at $298 \mathrm{~K}$, very close to our results $(91.1 \mathrm{~J} / \mathrm{mol} / \mathrm{K}$ without corrections, $77.42 \mathrm{~J} / \mathrm{mol} / \mathrm{K}$ with corrections). At lower temperature, Gonzalez et al. ${ }^{75,76}$ find $100 \mathrm{~J} / \mathrm{mol} / \mathrm{K}$ without corrections at $247 \mathrm{~K}$ and Saito et al. ${ }^{77,} 78$ find $96 \mathrm{~J} / \mathrm{mol} / \mathrm{K}$ without corrections at $250 \mathrm{~K}$. We find a $10 \%$ larger value: $110 \mathrm{~J} / \mathrm{mol} / \mathrm{K}$ before corrections at $250 \mathrm{~K}$. A more significant difference appears below $250 \mathrm{~K}$ as shown in Fig. 3 where the classical Molecular Dynamics (MD) data from Saito et al. ${ }^{78}$ are reproduced (dotted lines). A $25 \mathrm{~K}$ offset is observed between our $\mathrm{MC}$ results and $\mathrm{MD}$ data. Note that the $\mathrm{MC}$ data are obtained from the enthalpy fluctuations in the isothermalisobaric ensemble while the MD data are derived from the kinetic energy fluctuations in the microcanonical ensemble.

\section{Quantum effects}

The quantum corrections, due to the small mass of the hydrogen atoms, are taken into account so that the simulation results can be compared to experiments. Refined approaches can be found, using the full density of vibrational states from neutron scattering or from molecular dynamics, ${ }^{74,79}$ or full quantum mechanical treatment by path integral techniques. ${ }^{72}$, 80 However, such techniques are out of our scope, which is to obtain corrections based on a simple model. Our procedure will closely follow Owicki and Scheraga work. ${ }^{81}$ The contribution due to the zero-point energy is discarded: its contribution to the heat capacity arises only through its variation with temperature, which is negligible compared to other contributions to be considered now. This is due to the small dependence of absorption bands with temperature. ${ }^{82-84}$ The main contribution to the heat capacity comes from the molecular translations and rotations. Following Eisenberg and Kauzmann, ${ }^{85}$ these intermolecular vibrations can be seen as arising from a collection of quantum-mechanical harmonic oscillators. The corresponding intermolecular spectral density can be approximated either by two Debye spectra or by two Einstein spectra. The corresponding Debye and Einstein temperatures can be extracted from the experimental absorption bands for water in the far-IR. Owicki and Scheraga considered the two sets of frequencies: 200 and $700 \mathrm{~cm}^{-1}$ for Debye cutoff, and 115 and $450 \mathrm{~cm}^{-1}$ for Einstein frequencies. 81 The two models give similar results, as expected far from zero temperature. The experimental measurements of water IR spectra have since been improved, as well as its theoretical understanding, leading to the consideration of two peaks around 200 and $600 \mathrm{~cm}^{-1} .86-89$ With these values, the heat capacity obtained from Einstein's model at e.g. 173 and $298 \mathrm{~K}$ are 24.2 and $36.0 \mathrm{~J} / \mathrm{mol} / \mathrm{K}$ respectively. Note that we have neglected the small variations of the absorption bands with temperature to keep the model simple. Since we are interested in quantum corrections from our classical treatment by molecular dynamics, the simple harmonic oscillator approach is the most appropriate. The correction then simply consists in removing the classical $6 \mathrm{R}$ contribution from the intermolecular vibrational modes and replacing it with the heat capacity given by Einstein's model. The quantitative effect of these corrections is illustrated in Fig. 3 for the TIP4P/2005 model (the corrections are applied to the average data obtained from the descending and ascending curves). As can be seen, the quantum effects are quite important, with increasing amplitude at lower temperatures, as expected for quantum corrections. At 173 and $298 \mathrm{~K}$, the corrected heat capacities for the TIP4P/2005 model are 28 and $77.4 \mathrm{~J} / \mathrm{mol} / \mathrm{K}$. These values compare well with experimental data for ice $\mathrm{lh}$ at $173 \mathrm{~K}(24.8 \mathrm{~J} / \mathrm{mol} / \mathrm{K})$ and liquid water at $298 \mathrm{~K}(75.3 \mathrm{~J} / \mathrm{mol} / \mathrm{K})$, reported in Fig. 3. ${ }^{90-94}$ As can be seen, despite the simplicity of the approach, the quantum corrections considerably improve the agreement with experiments to within $3 \%$ at $298 \mathrm{~K}$ and $13 \%$ at $173 \mathrm{~K}$.

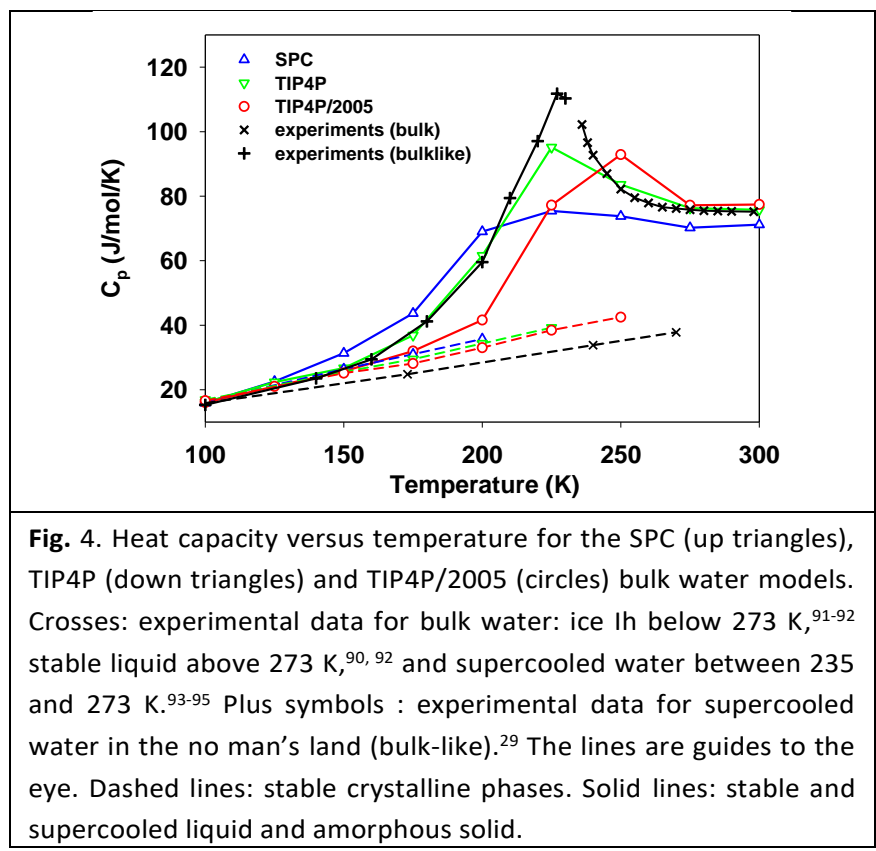

\section{Results and discussion}

\section{Bulk Water}

The heat capacity of bulk water has been calculated between 100 and $300 \mathrm{~K}$ for (i) the stable ice $\mathrm{lh}$, (ii) the stable liquid and (iii) the metastable amorphous solid or supercooled liquid (undistinguishable in our simulations). When the temperature is decreased, the system does not undergo the liquid-to-ice transition, even at low temperature. Instead, we observe a continuous transformation of the supercooled liquid into an amorphous solid. This was expected due to the large barriers to nucleate the crystalline ice phase. Conversely, the ice-to-liquid transition is observed for a sufficiently high temperature (between 225 and $270 \mathrm{~K}$, depending on the water model). The simulation results for the quantum corrected heat capacity of bulk water are shown in Fig. 4 for the three water models. For comparison, the experimental data ${ }^{29,90-95}$ are also given on the 
same figure. It is emphasized that these experimental data are for bulk water out of the no man's land (ice Ih below $273 \mathrm{~K}, 91,92$ liquid water above $273 \mathrm{~K}, 90,92$ supercooled water between 235 and $\left.273 \mathrm{~K}^{93-95}\right)$. The data in the supercooled no man's land are extrapolated from experiments in silica nanopores, and correspond to the contribution of internal water which is claimed to be close to bulk water by Mayurama et al. ${ }^{29}$

The three water models exhibit several common features. In particular, at low temperature $(100 \mathrm{~K})$, they give identical heat capacities within errors: $16.0 \pm 0.2 \mathrm{~J} / \mathrm{mol} / \mathrm{K}$ for the amorphous metastable solid, and $16.6 \pm 0.2 \mathrm{~J} / \mathrm{mol} / \mathrm{K}$ for the ice phase. These values are in excellent agreement with the experimental data $(15.3 \mathrm{~J} / \mathrm{mol} / \mathrm{K}){ }^{92}$ Furthermore, as can be seen, the heat capacity of the amorphous solid reaches that of the stable ice at $100 \mathrm{~K}$, reproducing the experimental behaviour. At high temperature $(300 \mathrm{~K})$ the three models give values between 71.2 and 77.4 $\mathrm{J} / \mathrm{mol} / \mathrm{K}$, which differ by less than $8 \%$. This difference is slightly larger than the uncertainties at that temperature: the SPC model slightly differs from the TIP4P and TIP4P/2005 models. However, the departure from experiments does not exceed $6 \%$ for SPC and 3\% for the TIP4P models. It is also observed that the three models exhibit a broad peak in the intermediate temperature region, in qualitative agreement with experimental observations.

The main differences between the models appear in the intermediate temperature region, where the heat capacity exhibits a maximum. The SPC model shows a broad peak between 200 and $250 \mathrm{~K}$ with a maximum at $75.4 \mathrm{~J} / \mathrm{mol} / \mathrm{K}$, i.e. $6 \%$ higher than the high temperature value. The TIP4P model has a narrower peak around $225 \mathrm{~K}$ with a maximum value at $95.2 \mathrm{~J} / \mathrm{mol} / \mathrm{K}$, i.e. $25 \%$ higher than at $300 \mathrm{~K}$. The TIP4P/2005 model also has a narrow peak, centred around $250 \mathrm{~K}$, with a maximum $(92.9 \mathrm{~J} / \mathrm{mol} / \mathrm{K}) 20 \%$ higher than at $300 \mathrm{~K}$. The experimental data show a strong peak around $225 \mathrm{~K}$, with a maximum $(112 \mathrm{~J} / \mathrm{mol} / \mathrm{K})$ which is $50 \%$ larger than at $300 \mathrm{~K}$.

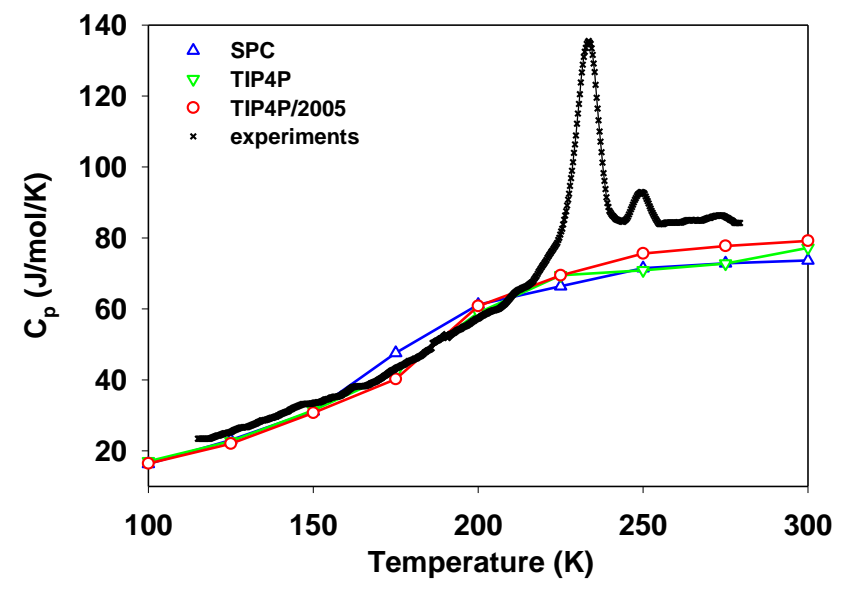

Fig. 5. Heat capacity versus temperature for the SPC (up triangles), TIP4P (down triangles) and TIP4P/2005 (circles) models of supercooled water thick films adsorbed on the smooth wall surface. Water confined in Vycor experiments (Ref [33]) (crosses) are reported for comparison. The lines are guides to the eye.

\section{Water films on smooth hydrophilic surface}

In experiments, bulk supercooled water is unstable between 150 and $230.6 \mathrm{~K}$. Therefore, most experiments use water confined in silica mesopores to perform measurements at low temperature. As in such material, the specific surface area is large, the contribution of the interaction of the confined/interfacial water molecules with the pore surface becomes predominant. The question of the influence of the silica wall thus arises. Furthermore, in most experiments, the pores are not necessarily saturated, and thus the film thickness could play a role. This is why the heat capacity of the water film is calculated for two different film thicknesses corresponding approximately to $26 \mu \mathrm{mol} / \mathrm{m}^{2}$ and $52 \mu \mathrm{mol} / \mathrm{m}^{2}$. The results obtained on the smooth hydrophilic surface are given in Fig. 5 and 6 . As can be seen, the curves exhibit almost monotonic variations with the temperature. At low and high temperatures (below $150 \mathrm{~K}$ and above $300 \mathrm{~K}$ ), the heat capacity is the same for both film thicknesses, and coincide with bulk values. On the other hand, in the intermediate region, small differences appear between the two film thicknesses for the SPC model: a small peak is observed at $225 \mathrm{~K}$ for the thin film. Except for this difference, the calculated heat capacities are essentially independent of the film thickness. Comparison with bulk data (Fig. 4) shows that the peak around $225 \mathrm{~K}$ has disappeared; however, the steep increase between 150 and $200 \mathrm{~K}$ remains unchanged.

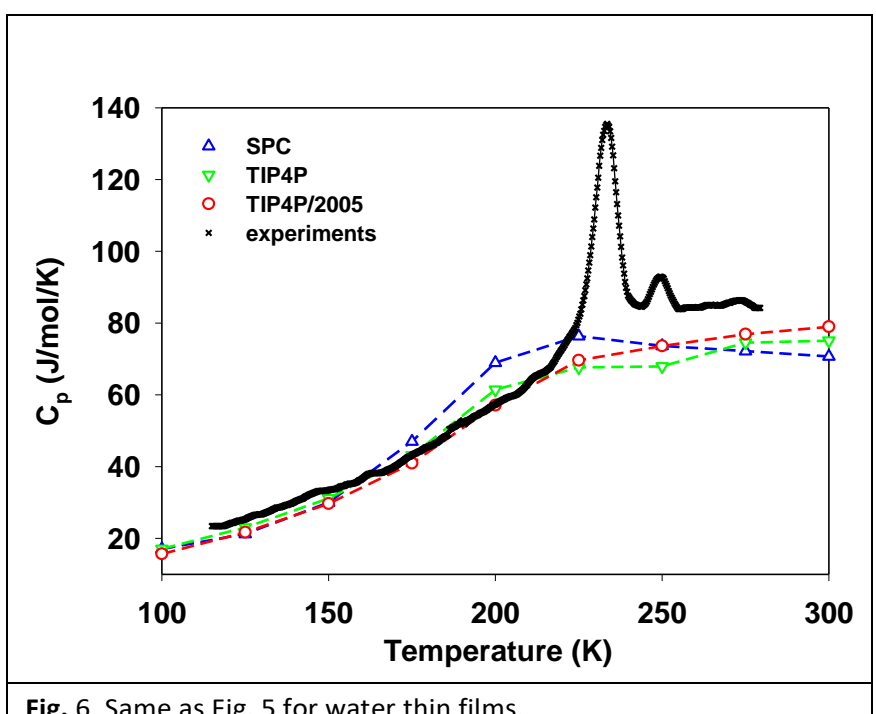

Fig. 6. Same as Fig. 5 for water thin films.

For comparison of simulations with experimental observations, we have reproduced on the same figure the data for a water film adsorbed in Vycor obtained by Tombari et al. ${ }^{33}$ More specifically, the experimental data correspond to $10 \mathrm{wt} \%$ (46\% filling), i.e. two water layers adsorbed on the silica surface. The heat capacity is measured upon decreasing the temperature, showing two peaks: the peak at $243 \mathrm{~K}$ corresponds to the delayed ice nucleation due to water confinement in nanopores (Gibbs-Thomson effect), while the peak at $233 \mathrm{~K}$ is associated with the supercooled adsorbed water. Comparison of 
simulations with experimental data should focus on the temperature range below $240 \mathrm{~K}$ since the calculations are performed on a flat surface and thus no Gibbs-Thomson effect is expected. As can be seen, the agreement in the low temperature region is quantitative. However, the simulations do not exhibit the sharp peak observed in Vycor at $233 \mathrm{~K}$.

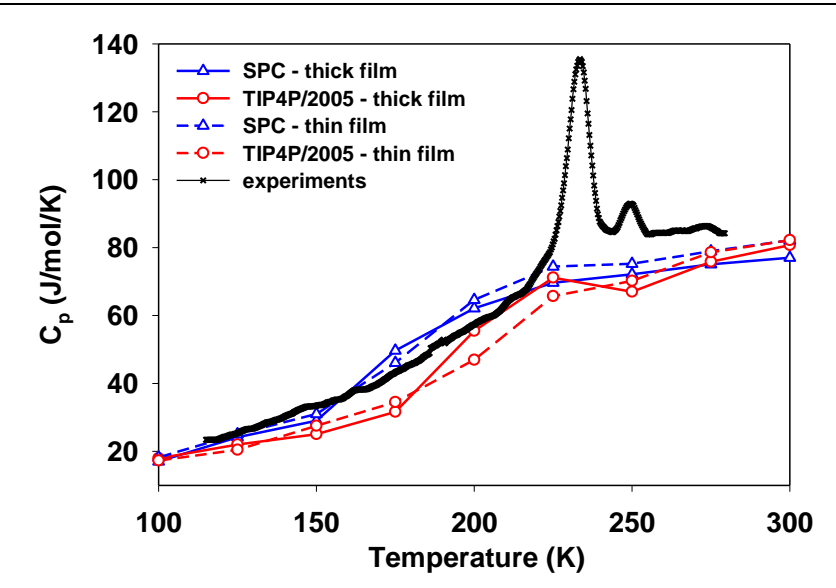

Fig. 7. Heat capacity versus temperature for the SPC (up triangles) and TIP4P/2005 (circles) water models, for thick (solid lines) and thin (dashed lines) films adsorbed on the anhydrous quartz (0001) surface. Water confined in Vycor experiments (Ref [33]) (crosses) are reported for comparison. The lines are guides to the eye.

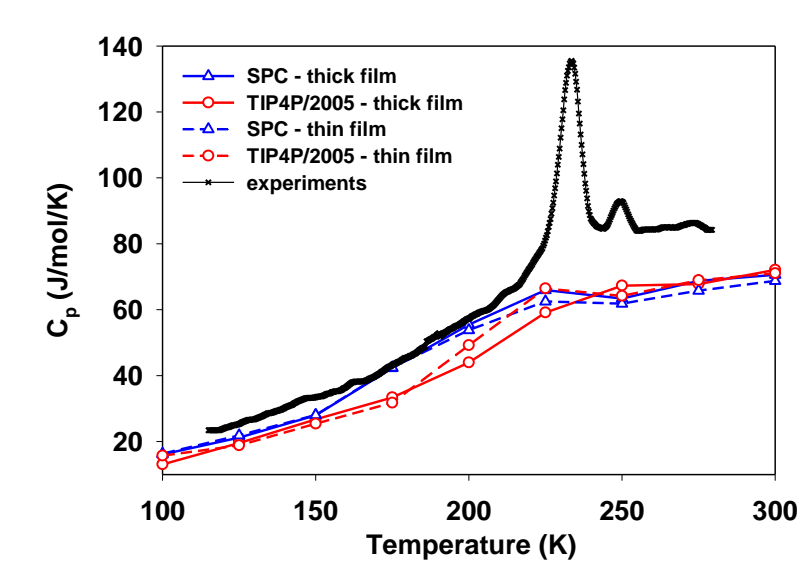

Fig. 8. Same as Fig. 7 for films adsorbed on the hydroxylated cristobalite surface.

\section{Water films on atomistic surfaces}

Two atomistic surfaces have been considered: the quartz (0001) and the cristobalite (001), two silica crystals. The quartz surface is perfectly stoichiometric $\mathrm{SiO}_{2}$, while the cristobalite surface is necessarily hydroxylated to compensate for the strong surface dipole. The heat capacity results for water films adsorbed on these surfaces are given in Figs 7 and 8 for the quartz and cristobalite respectively. The study has been limited to the SPC and TIP4P/2005 models, and two film thicknesses have been considered as previously $\left(26 \mu \mathrm{mol} / \mathrm{m}^{2}\right.$ and $\left.52 \mu \mathrm{mol} / \mathrm{m}^{2}\right)$. The first point to be noticed is that water on quartz bears many common features with water on smooth surfaces. At low and high temperatures (below $150 \mathrm{~K}$ and above $250 \mathrm{~K}$ ) the heat capacities of the two water models and film thicknesses are very close to the bulk and to the experimental values. All curves increase monotonously with temperature. The peak around 225 $\mathrm{K}$ observed in bulk water is replaced by a steep increase between 150 and $225 \mathrm{~K}$. One also observes that the film thickness has essentially no impact on the heat capacity. The only small difference is between the two water models: the onset of the steep increase of the heat capacity occurs $50 \mathrm{~K}$ lower for the SPC model.

The introduction of a hydroxylated surface does not change the results significantly except for a general lowering by more than $10 \%$ of the heat capacity values, in particular at high temperature (see Fig. 8). This reduction may originate in a disturbed dynamics at the interface due to the hydroxyls. The film thickness $\left(26 \mu \mathrm{mol} / \mathrm{m}^{2}\right.$ and $\left.52 \mu \mathrm{mol} / \mathrm{m}^{2}\right)$ has essentially no effect on the heat capacity. The steep increase around $200 \mathrm{~K}$ for TIP4P/2005 occurs $50 \mathrm{~K}$ lower for the SPC model.

\section{Discussion}

The three bulk water models exhibit a significant $C_{p}$ peak around $225 \mathrm{~K}$. One note however small differences between the models, that can be related to their intrinsic structure or mobility, which differs significantly between the models. The diffusion coefficient at ambient temperature is the highest for SPC $\left(4.3 \times 10^{-9} \mathrm{~m}^{2} / \mathrm{s}\right),{ }^{39}$ the lowest and closest to experiments for TIP4P/2005 $\left(2.08 \times 10^{-9} \mathrm{~m}^{2} / \mathrm{s}\right)^{40}$ and intermediate for TIP4P $\left(3.9 \times 10^{-9} \mathrm{~m}^{2} / \mathrm{s}\right) .^{42}$ The SPC and TIP4P models also significantly overestimate the tetrahedral structure of water, while TIP4P/2005 is closer to experiments. These properties could explain some differences in the position of the peak in $C_{p}$. For example, the dynamics can be correlated with the position of the peak: the faster the dynamics, the lower the temperature where the heat capacity increases rapidly. However, the best peak position agreement is not obtained for the TIP4P/2005 model, which, however, gives the best diffusion coefficient. The TIP4P and TIP4P/2005 models exhibit the highest peak, while the SPC peak is comparatively weak. This might be correlated with the structure of water, which is better reproduced by the TIP4P models. However, the differences do not exceed $10 \%$ in the position of the peak and $20 \%$ in amplitude. The existence of a heat capacity peak is thus related to generic properties of water models, in relation with its structure and dynamics, and its ability to form a supercooled phase.

The question of the influence of a surface has been examined by considering different situations, ranging from a structureless hydrophilic surface to atomistic pure $\mathrm{SiO}_{2}$ or hydroxylated silica surfaces. The main result regarding water films is the disappearance of the heat capacity peak. The curves are essentially independent of the film thickness, which is nevertheless in all cases quite small (few layers). Furthermore, a small reduction of the heat capacity is observed on the two most hydrophilic surfaces (the structureless and the hydroxylated ones). It is suggested that in these cases the water at the interface is highly structured, and its mobility partially 
hindered. Further investigation is required, using molecular dynamics algorithm for instance.

How does it compare with experiments? In real life, it is impossible for bulk water to reach the so-called no man's land in the supercooled region between 150 and $230.6 \mathrm{~K}$, because, upon cooling, supercooled water transforms into ice below $230.6 \mathrm{~K}, 21$ and, upon heating, amorphous solid water spontaneously crystallizes above $150 \mathrm{~K}$. Experiments are thus performed on water confined in nanoporous materials, which hinder the ice nucleation. ${ }^{26,28,29}$ The question thus arises to know whether the presence of an interface strongly affects the intrinsic properties of water. Experiments show a dependence of the heat capacity measurements both on pore size and pore filling. ${ }^{28,30,33}$ Tombari et al. show that the effect of pore filling is to enhance the $C_{p}$ peak intensity while it shifts its position to higher temperatures (from $250 \mathrm{~K}$ at $27 \%$ filling to $260 \mathrm{~K}$ at $100 \%$ filling). ${ }^{33}$ The authors underline a strong similarity with Oguni $e t$ al. ${ }^{30}$ results on the dependence with pore size. This broad endothermic peak observed upon sample heating is attributed by the authors to the melting of ice in the pores. ${ }^{28,30,33}$ Tombari et al. ${ }^{33}$ have compared the calorimetric curves upon heating and cooling. They show that cooling curves are quite different from heating ones, and support the hypothesis of ice crystallization for pore filling larger than $46 \%$ (1-2 water layers), with a considerable hysteresis in the corresponding temperature peaks. ${ }^{33}$ Takamuku et al. ${ }^{28}$ arrived at similar conclusions including both the effect of pore filling and pore size. More interestingly, these authors observe the appearance of a peak at $233 \mathrm{~K}$ upon cooling, when the amount of water or the pore size are small enough so that the ice crystallization is impeded. Oguni et al..$^{30}$ have also observed a small hump in the $C_{p}$ curves when the pores are small enough $(1.1 \mathrm{~nm})$ to prevent water crystallization down to $80 \mathrm{~K}$. They also show that the pores cannot be chosen too small, otherwise water cannot develop bulk-like clusters. ${ }^{31}$ Maruyama et al. ${ }^{29}$ have also considered water confined in silica gels in pores small enough to prevent ice formation but large enough to allow the formation of bulklike clusters $(3 \mathrm{~nm})$. They interpret the data as being mainly due to the internal bulk-like supercooled water, and, subtracting the interfacial contribution, they show that the heat capacity exhibits a peak around $227 \mathrm{~K}$, in agreement with Tombari and Oguni observations. ${ }^{30,} 33$ Maruyama et al. ${ }^{29}$ and Oguni et al..$^{30}$ outline the smooth connection of this $C_{p}$ peak around $227 \mathrm{~K}$ with the bulk supercooled water data above $235 \mathrm{~K}$, and suggest that the thermodynamic properties of this internal water are close to those of bulk supercooled water. Our bulk water simulation results are thus compared with these experimental data, and exhibit a rather good agreement.

\section{Conclusion}

The heat capacity of supercooled water has been calculated for three water models: SPC, TIP4P and TIP4P/2005. Two situations have been considered: bulk water and thin films adsorbed on hydrophilic surfaces. A simple model for quantum corrections has been applied. It enables a good quantitative agreement with experiments at high $(>275 \mathrm{~K})$ and low $(<150 \mathrm{~K})$ temperatures.

For intermediate temperatures, the three water models exhibit a $C_{p}$ peak, in agreement with experiments. ${ }^{29}$ However, the deconvolution of experimental data obtained in small pores from interfacial contributions relies on the hypothesis of a twostate model where the $C_{p}$ peak could clearly be decomposed into two contributions from bulk-like water in the core of the pore and interfacial water. This scenario seems to be supported by our simulations, which show that thin films of adsorbed water do not contribute to the $C_{p}$ peak, whatever the hydrophilic surface (structureless vs atomistic, anhydrous vs hydroxylated). It is however poorly supported by experiments: (i) the range of pore size is tedious where the contribution of the internal supercooled water is maximal, and seems to depend on the silica material, (ii) an analogy can be drawn between the effect of pore size and pore filling, and (iii) one observes anomalies in interfacial water or water confined in very small pores. $22,26-31,33,35,96 \mathrm{It}$ is believed that the surface properties of the Vycor or silica gel play a fundamental role. These highly hydroxylated, disordered amorphous silica surfaces are able to establish a high degree of hydrogenbonding with the adsorbed water, with very specific dynamics at the interface that could explain the different anomalies observed for interfacial water. ${ }^{35}$ Takamuku et al. outlined the importance of the silanol groups in the dynamics of water molecules in the pores. ${ }^{28}$ Our still crude model of hydrophilic surface is unable to catch these phenomena, and deserve further studies. Several directions are privileged: (i) considering more realistic amorphous silica surfaces, with a degree of hydroxylation closer to Vycor surfaces $\left(16 \mathrm{OH} / \mathrm{nm}^{2}\right)^{22}$ and possibly including disorder, ${ }^{54-56}$ which favors the formation of clusters that are believed to play an important role; (ii) studying the effect of the geometry of the water/silica interface, since this is known to influence water properties, in particular in small pores; (iii) improving the water-silica interaction model, since it has been shown that the hydrophilic properties of silica are much more sensitive to the potential than the surface hydroxyl density; ${ }^{44,97}$ and, (iv) improving the silica interatomic potential, in particular regarding the dynamics of the silanol groups interacting with water.

\section{Conflicts of interest}

"There are no conflicts to declare".

\section{Acknowledgements}

The authors acknowledge financial support from ANR-17-CE30002-04.

\section{Notes and references}

1. R. J. Speedy, J. Phys. Chem., 1982, 86, 982-991. 
2. P. H. Poole, F. Sciortino, U. Essmann and H. E. Stanley, Nature, 1992, 360, 324-328.

3. O. Mishima and H. E. Stanley, Nature, 1998, 396, 329-335.

4. P. Gallo, M. Rovere and E. Spohr, Phys. Rev. Lett., 2000, 85, 43174320.

5. P. G. Debenedetti and H. E. Stanley, Physics Today, 2003, 56, 4046.

6. L. Liu, S.-H. Chen, A. Faraone, C.-W. Yen and C.-Y. Mou, Phys. Rev. Lett., 2005, 95, 117802.

7. F. Mallamace, M. Broccio, C. Corsaro, A. Faraone, L. Liu, C.-Y. Mou and S.-H. Chen, J. Phys.: Condens. Matter, 2006, 18, S2285-S2297.

8. G. P. Johari, J. Chem. Phys., 2009, 130, 124518.

9. J. Swenson and S. Cerveny, J. Phys.: Condens. Matter, 2014, 27, 033102.

10. M. Sattig and M. Vogel, J. Phys. Chem. Lett., 2014, 5, 174-178.

11. F. Caupin, J. Non-Cryst. Solids, 2015, 407, 441-448.

12. A. Nilsson and L. G. M. Pettersson, Nat. Commun., 2015, 6, 8998.

13. S. Cerveny, F. Mallamace, J. Swenson, M. Vogel and L. Xu, Chem. Rev., 2016, 116, 7608-7625.

14. V. De Michele, G. Romanelli and A. Cupane, Front. Phys., 2017, 13, 138205.

15. K. H. Kim, A. Späh, H. Pathak, F. Perakis, D. Mariedahl, K. AmannWinkel, J. A. Sellberg, J. H. Lee, S. Kim, J. Park, K. H. Nam, T. Katayama and A. Nilsson, Science, 2017, 358, 1589.

16. C. Lederle, M. Sattig and M. Vogel, J. Phys. Chem. C, 2018, 122, 15427-15434.

17. F. Caupin and M. A. Anisimov, J. Chem. Phys., 2019, 151, 034503.

18. R. Neffati, P. Judeinstein and J. Rault, J. Phys.: Condens. Matter, 2020, 32, 465101.

19. F. Mallamace, C. Corsaro, D. Mallamace, E. Fazio, S.-H. Chen and A. Cupane, Int. J. Mol. Sci., 2020, 21, 622.

20. R. J. Speedy and C. A. Angell, J. Chem. Phys., 1976, 65, 851-858.

21. C. Goy, M. A. C. Potenza, S. Dedera, M. Tomut, E. Guillerm, A. Kalinin, K.-O. Voss, A. Schottelius, N. Petridis, A. Prosvetov, G. Tejeda, J. M. Fernández, C. Trautmann, F. Caupin, U. Glasmacher and R. E. Grisenti, Phys. Rev. Lett., 2018, 120, 015501.

22. J.-M. Zanotti, M.-C. Bellissent-Funel and S. H. Chen, Europhys. Lett., 2005, 71, 91.

23. S. Kittaka, S. Takahara, H. Matsumoto, Y. Wada, T. J. Satoh and T. Yamaguchi, J. Chem. Phys., 2013, 138, 204714.

24. E. Stefanutti, L. E. Bove, F. G. Alabarse, G. Lelong, F. Bruni and M. A. Ricci, J. Chem. Phys., 2019, 150, 224504.

25. M. Weigler, M. Brodrecht, G. Buntkowsky and M. Vogel, J. Phys. Chem. B, 2019, 123, 2123-2134.

26. J.-M. Zanotti, M.-C. Bellissent-Funel and S.-H. Chen, Phys. Rev. E, 1999, 59, 3084-3093.

27. J.-M. Zanotti, M.-C. Bellissent-Funel, S.-H. Chen and A. I. Kolesnikov, J. Phys.: Condens. Matter, 2006, 18, S2299-S2304.

28. T. Takamuku, M. Yamagami, H. Wakita, Y. Masuda and T. Yamaguchi, J. Phys. Chem. B, 1997, 101, 5730-5739.

29. S. Maruyama, K. Wakabayashi and M. Oguni, AIP Conf. Proc., 2004, 708, 675-676.

30. M. Oguni, S. Maruyama, K. Wakabayashi and A. Nagoe, Chem. Asian J., 2007, 2, 514-520.

31. M. Oguni, Y. Kanke and S. Namba, AIP Conf. Proc., 2008, 982, 3438.

32. S. Jähnert, F. Vaca Chávez, G. E. Schaumann, A. Schreiber, M. Schönhoff and G. H. Findenegg, Phys. Chem. Chem. Phys., 2008, 10, 6039-6051.

33. E. Tombari, C. Ferrari, G. Salvetti and G. P. Johari, Thermochim Acta, 2009, 492, 37-44.

34. E. Tombari and G. P. Johari, J. Chem. Phys., 2013, 139, 064507.
35. J.-M. Zanotti, P. Judeinstein, S. Dalla-Bernardina, G. Creff, J.-B. Brubach, P. Roy, M. Bonetti, J. Ollivier, D. Sakellariou and M.-C. Bellissent-Funel, Sci. Rep., 2016, 6, 25938.

36. N. Giovambattista, P. J. Rossky and P. G. Debenedetti, Annu. Rev. Phys. Chem., 2012, 63, 179-200.

37. B. Guillot, J. Mol. Liquids, 2002, 101, 219-260.

38. J. F. Ouyang and R. P. A. Bettens, Chimia, 2015, 69, 104-111.

39. H. J. C. Berendsen, J. P. M. Postma, W. F. van Gunsteren and J. Hermans, in Intermolecular Forces, B. Pullman, Dordrecht: Reidel, 1981, p. 331.

40. W. L. Jorgensen, J. Chandrasekhar, J. D. Madura, R. W. Impey and M. L. Klein, J. Chem. Phys., 1983, 79, 926-935.

41. W. L. Jorgensen and C. Jenson, J. Comp. Chem., 1998, 19, 11791186.

42. J. L. F. Abascal and C. Vega, J. Chem. Phys., 2005, 123, 234505.

43. I. Brovchenko, A. Geiger and A. Oleinikova, Phys. Chem. Chem. Phys., 2001, 3, 1567-1569.

44. J. Puibasset and R. J.-M. Pellenq, J. Chem. Phys., 2003, 118, 56135622.

45. J. Puibasset and R. J.-M. Pellenq, J. Phys.: Condens. Matter, 2004, 16, S5329-S5343.

46. J. Puibasset, J. Phys. Chem. B, 2005, 109, 8185-8194.

47. J. Puibasset, J. Chem. Phys., 2005, 122, 134710.

48. C. Mischler, J. Horbach, W. Kob and K. Binder, J. Phys.: Condens. Matter, 2005, 17, 4005-4013.

49. A. Malani, K. G. Ayappa and S. Murad, J. Phys. Chem. B, 2009, 113, 13825-13839.

50. P. A. Bonnaud, B. Coasne and R. J. M. Pellenq, J. Phys.: Condens. Matter, 2010, 22, 284110.

51. K. Yamashita and H. Daiguji, J. Phys. Chem. C, 2013, 117, 20842095.

52. K. Yamashita, K. Kashiwagi, A. Agrawal and H. Daiguji, Mol. Phys., 2017, 115, 328-342.

53. B. Kuchta, P. Llewellyn, R. Denoyel and L. Firlej, Low Temp. Phys., 2003, 29, 880-882.

54. J. Puibasset, J. Phys. Chem. B, 2005, 109, 4700-4706.

55. J. Puibasset, J. Chem. Phys., 2006, 125, 074707.

56. B. Kuchta, L. Firlej, M. Marzec and P. Boulet, Langmuir, 2008, 24, 4013-4019.

57. Z. Feng, X. Zhang and W. Wang, Phys. Rev. E, 2008, 77, 051603.

58. D. Argyris, D. R. Cole and A. Striolo, J. Phys. Chem. C, 2009, 113, 19591-19600.

59. C. Vega, J. L. F. Abascal, M. M. Conde and J. L. Aragones, Faraday Discussions, 2009, 141, 251-276.

60. W. L. Jorgensen, Chem. Phys. Lett., 1982, 92, 405-410.

61. A. Bródka and T. W. Zerda, J. Chem. Phys., 1996, 104, 6319-6326.

62. J. Puibasset and R. J.-M. Pellenq, Phys. Chem. Chem. Phys., 2004, 6, 1933-1937.

63. J. Puibasset, P. Judeinstein and J. M. Zanotti, Mol. Simul., 2018, 45, 462-465.

64. N. H. de Leeuw, F. M. Higgins and S. C. Parker, J. Phys. Chem. B, 1999, 103, 1270-1277.

65. Y.-W. Chen, C. Cao and H.-P. Cheng, Appl. Phys. Lett., 2008, 93, 181911.

66. L. T. Zhuravlev, Colloids Surf. A: Physicochem. Eng. Aspects, 2000, 173, 1-38.

67. J. Puibasset and R. J.-M. Pellenq, J. Chem. Phys., 2003, 119, 92269232.

68. M. P. Allen and D. J. Tildesley, Computer Simulation of Liquids, Clarendon Press, Oxford, 1987.

69. W. L. Jorgensen and J. D. Madura, Mol. Phys., 1985, 56, 13811392. 
70. W. L. Jorgensen and J. Tirado-Rives, Proc. Natl. Acad. Sci. U. S. A., 2005, 102, 6665.

71. Y. Wu, H. L. Tepper and G. A. Voth, J. Chem. Phys., 2006, 124, 024503.

72. C. Vega, M. M. Conde, C. McBride, J. L. F. Abascal, E. G. Noya, R. Ramirez and L. M. Sesé, J. Chem. Phys., 2010, 132, 046101.

73. C. Vega and J. L. F. Abascal, Phys. Chem. Chem. Phys., 2011, 13, 19663-19688.

74. T. A. Pascal, D. Schärf, Y. Jung and T. D. Kühne, J. Chem. Phys., 2012, 137, 244507.

75. M. A. González, C. Valeriani, F. Caupin and J. L. F. Abascal, J. Chem. Phys., 2016, 145, 054505.

76. J. W. Biddle, R. S. Singh, E. M. Sparano, F. Ricci, M. A. González, C. Valeriani, J. L. F. Abascal, P. G. Debenedetti, M. A. Anisimov and F. Caupin, J. Chem. Phys., 2017, 146, 034502.

77. S. Saito, I. Ohmine and B. Bagchi, J. Chem. Phys., 2013, 138, 094503.

78. S. Saito and B. Bagchi, J. Chem. Phys., 2019, 150, 054502.

79. D. D. Klug, E. Whalley, E. C. Svensson, J. H. Root and V. F. Sears, Phys. Rev. B, 1991, 44, 841-844.

80. M. Shiga and W. Shinoda, J. Chem. Phys., 2005, 123, 134502.

81. J. C. Owicki and H. A. Scheraga, J. Am. Chem. Soc., 1977, 99, 74037412.

82. H. W. Horn, W. C. Swope, J. W. Pitera, J. D. Madura, T. J. Dick, G. L. Hura and T. Head-Gordon, J. Chem. Phys., 2004, 120, 96659678.

83. C. Medcraft, D. McNaughton, C. D. Thompson, D. Appadoo, S. Bauerecker and E. G. Robertson, Astrophys. J., 2012, 758, 17.

84. C. Medcraft, D. McNaughton, C. D. Thompson, D. R. T. Appadoo, S. Bauerecker and E. G. Robertson, Phys. Chem. Chem. Phys., 2013, 15, 3630-3639.

85. D. Eisenberg and W. Kauzmann, The Structure and Properties of Water, Oxford University Press, London, 1969.

86. D. A. Draegert, N. W. B. Stone, B. Curnutte and D. Williams, J. Opt. Soc. Am., 1966, 56, 64-69.

87. J. E. Bertie and Z. Lan, Appl. Spectrosc., 1996, 50, 1047-1057.

88. P. L. Silvestrelli, M. Bernasconi and M. Parrinello, Chem. Phys. Lett., 1997, 277, 478-482.

89. H. Liu, Y. Wang and J. M. Bowman, J. Chem. Phys., 2015, 142, 194502.

90. G. S. Kell, J. Chem. Eng. Data, 1975, 20, 97.

91. R. Feistel and W. Wagner, J. Phys. Chem. Ref. Data, 2006, 35, 1021-1047.

92. CRC Handbook of Chemistry and Physics, 86th Edition Edited by David R. Lide (National Institute of Standards and Technology), American Chemical Society, 2006.

93. E. Tombari, C. Ferrari and G. Salvetti, Chem. Phys. Lett. 1999, 300, 749-751.

94. V. P. Voronov, V. E. Podnek, M. A. Anisimov, J. Phys.: Conf. Ser. 2019, 1385, 012008.

95. C. A. Angell, M. Oguni and W. J. Sichina, J. Phys. Chem., 1982, 86, 998-1002.

96. E. Tombari, G. Salvetti, C. Ferrari and G. P. Johari, J. Chem. Phys., 2005, 122, 104712

97. E. Pafong, J. Geske and B. Drossel, J. Chem. Phys., 2016, 145, 114901. 